\title{
Diagnostic feature of tuberculous peritonitis in patients with cirrhosis: A matched case-control study
}

\author{
HAI-JUN HUANG ${ }^{1}$, JIN YANG $^{2,3}$, YI-CHENG HUANG ${ }^{1}$, HONG-YING PAN ${ }^{1}$, \\ $\mathrm{HONG}_{\mathrm{WANG}}{ }^{1}$ and $\mathrm{ZHUO}-\mathrm{CHAO} \mathrm{REN}^{4}$ \\ ${ }^{1}$ Department of Infectious Disease, Zhejiang Provincial People's Hospital, Hangzhou, Zheijiang 310014; \\ ${ }^{2}$ Department of Medicine, Blood Center of Zhejiang Province, Hangzhou, Zheijiang 310061; \\ ${ }^{3}$ Transform Medicine Center, Affiliated Hospital of Hangzhou Normal University, Hangzhou, Zheijiang 310015; \\ ${ }^{4}$ Department of Respiratory Medicine, Zhejiang Provincial People's Hospital, Hangzhou, Zheijiang 310014, P.R. China
}

Received September 26, 2013; Accepted January 30, 2014

DOI: $10.3892 / e t m .2014 .1538$

\begin{abstract}
The aim of the present study was to compare the clinical characteristics of tuberculous peritonitis (TBP) and spontaneous bacterial peritonitis (SBP) in patients with cirrhosis. A retrospective, matched case-control study was conducted consisting of 12 patients with cirrhosis diagnosed with TBP between 2008 and 2011. Control subjects were patients with SBP. Clinical features and laboratory data were analyzed. Compared with SBP, TBP in patients with cirrhosis was frequently associated with extraperitoneal tuberculosis ( 25 vs. $0 \%)$, a more insidious onset $(39.67 \pm 30.00$ vs. $21.60 \pm 21.50$ days; $\mathrm{P}<0.05)$, Child-Pugh classification $\mathrm{B}$ at onset $(67$ vs. $32 \% ; \mathrm{P}<0.05)$ and lymphopenia $(0.67 \pm 0.22$ vs. $\left.1.19 \pm 0.41 \times 10^{9} / 1 ; \mathrm{P}<0.01\right)$. Patients with TBP tended to have lymphocytic predominance in the peritoneal fluid (92\%), while patients with SBP tended to have neutrophil predominance (68\%). Compared with the SBP group, the TBP group had significantly higher ascitic protein, adenosine deaminase (ADA) and lactate dehydrogenase (LDH) levels. Ascitic protein levels were $>25 \mathrm{~g} / \mathrm{l}$ in 9 patients (75\%) in the TBP group and in 2 patients (8\%) in the SBP group; ascitic ADA activity levels were $>27 \mathrm{U} / 1$ in 8 patients (67\%) in the TBP group, but were not $>27 \mathrm{U} / 1$ in any of the patients in the SBP group; ascitic LDH levels were $>90 \mathrm{U} / 1$ in 10 patients (83\%) in the TBP group and 5 patients (20\%) in the SBP group. Therefore, the results of the present study indicate that TBP should be considered in cirrhotic patients with relevant clinical manifestations and characteristics of laboratory observations.
\end{abstract}

Correspondence to: Professor Hong-Ying Pan, Department of Infectious Disease, Zhejiang Provincial People's Hospital, 158 Shangtang Road, Hangzhou, Zheijiang 310014, P.R. China E-mail: panhongying163@126.com

Key words: tuberculous peritonitis, spontaneous bacterial peritonitis, liver cirrhosis

\section{Introduction}

In previous years, there has been a global increase in the incidence of tuberculosis (TB), along with the prevalence of acquired immunodeficiency syndrome, and the emergence of multidrug-resistant strains. Tuberculous peritonitis (TBP) is primarily caused by hematogenous spread and rarely results from the contagious spread of an infected bowel or fallopian tubes $(1,2)$. It is estimated that TBP represents $4-10 \%$ of all extrapulmonary TB cases $(3,4)$.

Diagnosis of TBP is difficult since the clinical features are nonspecific and ascitic fluid may contain few tubercle bacilli that can neither be observed nor cultured. Undiagnosed and untreated TBP results in a mortality rate of $50-60 \%$ (5); however, the disease is usually curable when treated properly. Patients with liver cirrhosis are at an increased risk of developing TBP $(6,7)$. TBP in cirrhotic patients can mimic spontaneous bacterial peritonitis (SBP) and is frequently not considered in differential diagnosis, resulting in delayed diagnosis and even mortality (7). Awareness of the clinical features of TBP in patients with cirrhosis is crucial for improving diagnostic accuracy and survival. However, the study of TBP characteristics in patients with cirrhosis, compared with those of SBP, is limited (8). Therefore, a retrospective, matched case-control study was performed to compare the clinical characteristics of TBP and SBP in patients with cirrhosis.

\section{Materials and methods}

Patient selection. In this retrospective study, the hospital records of 12 patients with cirrhosis who were diagnosed with TBP in the Zhejiang Provincial People's Hospital (Hangzhou, China) between 2008 and 2011 were reviewed. For the purpose of comparison, 25 patients with definite SBP were selected that matched the TBP patients in age and gender during the same period. The study was approved by the Human Ethics Committee of Zhejiang Provincial People's Hospital. Written informed consent was obtained from the patient's family.

Methods. The diagnosis of liver cirrhosis was confirmed by clinical observations, image analysis or the presence of 
Table I. Demographic and clinical characteristics of the study population.

\begin{tabular}{|c|c|c|c|}
\hline Characteristics & $\mathrm{TBP}(\mathrm{n}=12)$ & $\operatorname{SBP}(n=25)$ & P-value \\
\hline Age, years & $58.75 \pm 12.66^{\mathrm{a}}$ & $57.84 \pm 14.19^{\mathrm{a}}$ & NS \\
\hline Male, n (\%) & $8(67)$ & $16(64)$ & NS \\
\hline \multicolumn{4}{|l|}{ Etiology of cirrhosis, n (\%) } \\
\hline Hepatitis B virus & $8(67)$ & $17(68)$ & NS \\
\hline Hepatitis $\mathrm{C}$ virus & 0 & $1(4)$ & NS \\
\hline Alcohol & $3(25)$ & $3(12)$ & NS \\
\hline Schistosome & $1(8)$ & $2(8)$ & NS \\
\hline Biliary & 0 & $2(8)$ & NS \\
\hline Child-Pugh class, n (\%) & & & $<0.05$ \\
\hline $\mathrm{B}$ & $8(67)$ & $8(32)$ & \\
\hline $\mathrm{C}$ & $4(33)$ & $17(68)$ & \\
\hline Tuberculosis at other site, $\mathrm{n}(\%)$ & $3(25)$ & $0(0)$ & $<0.01$ \\
\hline Duration of symptoms before presentation, days & $39.67 \pm 30.00^{\mathrm{a}}$ & $21.60 \pm 21.50^{\mathrm{a}}$ & $<0.05$ \\
\hline \multicolumn{4}{|l|}{ Initial symptoms, n (\%) } \\
\hline Abdominal distension & $11(92)$ & $23(92)$ & NS \\
\hline Fever & $6(50)$ & $8(32)$ & NS \\
\hline Abdominal pain & $5(42)$ & $9(36)$ & NS \\
\hline Diarrhea & $3(25)$ & $6(24)$ & NS \\
\hline
\end{tabular}

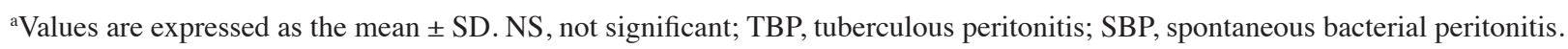

esophagogastric varices. The severity of liver cirrhosis was graded according to the Child-Pugh classification. Patients with human immunodeficiency virus coinfection or hepatocellular carcinoma were excluded.

All 12 cirrhotic patients with compatible symptoms, including fever, abdominal pain and distention, were diagnosed with TBP if one or more of the following criteria was met: i) Positive culture of Mycobacterium tuberculosis from ascites; ii) positive detection of acid-fast bacilli in ascites; iii) demonstration of caseating granulomata in histological examination of peritoneal biopsy specimens; iv) positive detection of Mycobacterium tuberculosis in ascites after polymerase chain reaction (PCR); and v) response to antituberculous therapy.

All 25 patients with cirrhosis and clinical manifestations of SBP were diagnosed with definite SBP, defined as SBP caused by one monobacteria (culture was positive for ascites) and a polymorphonuclear leukocyte count in the ascitic fluid of $\geq 250$ cells $/ \mu 1$. Patients with suspected secondary peritonitis were excluded, as discussed by Rimola et al (9).

Patient demographics, clinical manifestations, presence of extraperitoneal tuberculosis, hematological data, ascetic fluid analysis and the culture of ascites for bacteria were recorded. The culture of biopsies or ascitic fluid for Mycobacterium tuberculosis was not performed.

Statistical analyses. Proportions were compared using the $\chi^{2}$ test or a two-tailed Fisher's exact test. Continuous variables were compared using the Student's t-test or the Mann-Whitney U test. Statistical analyses were performed using SPSS software, version 12.0 (SPSS, Inc., Chicago,
IL, USA). $\mathrm{P}<0.05$ was considered to indicate a statistically significant difference.

\section{Results}

Demographic and clinical manifestations. Demographic and clinical characteristics of the 37 participants are shown in Table I. In the TBP group, three cases demonstrated caseating granulomata following histological assessment, two cases showed a positive culture of Mycobacterium tuberculosis, two cases detected positive for acid-fast bacilli and in the remaining five patients, diagnosis was based on the positive result of PCR and the response to antituberculous therapy. Of the 25 patients in the SBP group, 12 cases were infected with Escherichia coli, seven cases were infected with Klebsiella species, three cases were infected with Streptococcus species, two cases were infected with Staphylococcus species and one case was infected with Aeromonas species. The frequency of Child-Pugh class B was significantly higher in the TBP group when compared with the SBP group [8/12 patients $(67 \%)$ vs. $8 / 25$ patients $(32 \%)$; $\mathrm{P}<0.05]$. Three cases $(25 \%)$ in the TBP group exhibited pulmonary TB, but no case was identified in the SBP group. A statistically significant increase in the median duration of symptoms prior to presentation was observed in the TBP group (39.67 \pm 30.00 vs. 21.60 \pm 21.50 days; $\mathrm{P}<0.05)$. There were no other statistically significant differences between the groups with regard to age, gender, etiology of cirrhosis and initial clinical symptoms.

Laboratory observations. Laboratory observations are summarized in Table II and Fig. 1. The mean peripheral 
Table II. Laboratory observations of patients with TBP and SBP.

\begin{tabular}{|c|c|c|c|}
\hline Parameters & $\operatorname{TBP}(n=12)$ & $\operatorname{SBP}(n=25)$ & P-value \\
\hline \multicolumn{4}{|c|}{ Hematological observations upon admission } \\
\hline White cell count, $10^{9} / 1$ & $4.83 \pm 1.45^{\mathrm{a}}$ & $7.58 \pm 5.78^{\mathrm{a}}$ & NS \\
\hline Lymphocyte, $10^{9} / 1$ & $0.67 \pm 0.22^{\mathrm{a}}$ & $1.19 \pm 0.41^{\mathrm{a}}$ & $<0.01$ \\
\hline Protein, g/l & $71.19 \pm 7.28^{\mathrm{a}}$ & $63.90 \pm 8.92^{\mathrm{a}}$ & $<0.05$ \\
\hline Albumin, g/l & $31.62 \pm 5.08^{\mathrm{a}}$ & $27.48 \pm 4.16^{\mathrm{a}}$ & $<0.05$ \\
\hline $\mathrm{CA}-125, \mathrm{U} / \mathrm{ml}$ & $594 \pm 504^{\mathrm{a}}$ & $439 \pm 340^{\mathrm{a}}$ & NS \\
\hline \multicolumn{4}{|c|}{ Ascitic fluid observations upon admission } \\
\hline White cell count, $\mu 1$ & $1840 \pm 1503^{\mathrm{a}}$ & $1390 \pm 1912^{\mathrm{a}}$ & NS \\
\hline Lymphocyte predominant, n (\%) & $11(92)$ & $1(4)$ & $<0.01$ \\
\hline Neutrophil predominant, $\mathrm{n}(\%)$ & 0 & $17(68)$ & $<0.01$ \\
\hline Monocyte predominant, $\mathrm{n}(\%)$ & 0 & $4(16)$ & NS \\
\hline Equivocal, n (\%) & $1(8)$ & $3(12)$ & NS \\
\hline Protein, g/dl & $38.50 \pm 11.96^{\mathrm{a}}$ & $12.94 \pm 9.16^{\mathrm{a}}$ & 0 \\
\hline$>25 \mathrm{~g} / \mathrm{l}, \mathrm{n}(\%)$ & $9(75)$ & $2(8)$ & 0 \\
\hline \multicolumn{4}{|l|}{ SAAG } \\
\hline$\geq 11 \mathrm{~g} / 1$ & $10(83)$ & $22(88)$ & NS \\
\hline $\mathrm{ADA}, \mathrm{U} / 1$ & $34.67 \pm 17.54^{\mathrm{a}}$ & $4.32 \pm 2.25^{\mathrm{a}}$ & 0 \\
\hline$\geq 30 \mathrm{U} / 1, \mathrm{n}(\%)$ & $8(67)$ & 0 & 0 \\
\hline $\mathrm{LDH}, \mathrm{U} / 1$ & $203.83 \pm 150.55^{\mathrm{a}}$ & $57.44 \pm 48.06^{\mathrm{a}}$ & 0 \\
\hline$\geq 90 \mathrm{U} / 1$ & $10(83)$ & $5(20)$ & 0 \\
\hline
\end{tabular}

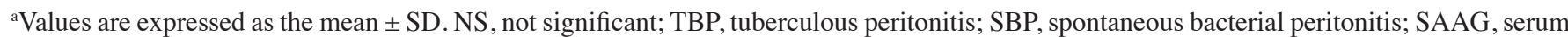
ascites albumin gradient; ADA, adenosine deaminase; LDH, lactate dehydrogenase; CA-125, cancer antigen 125.

A

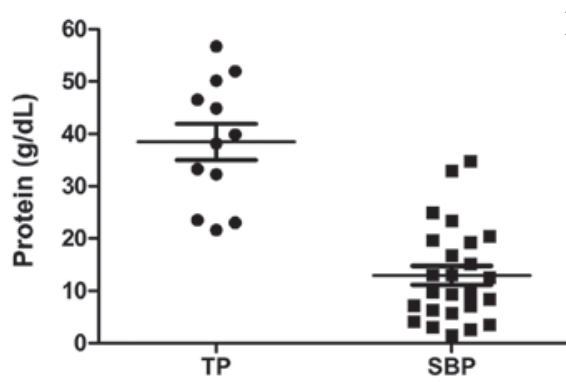

C

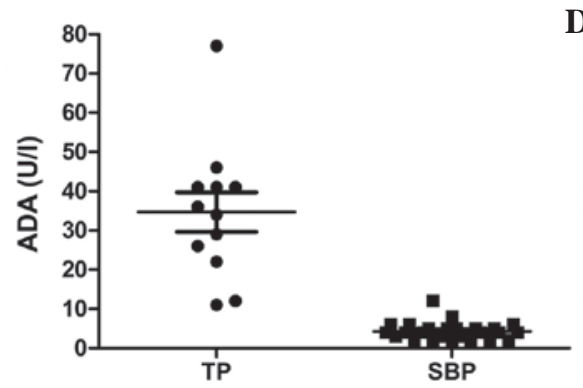

B
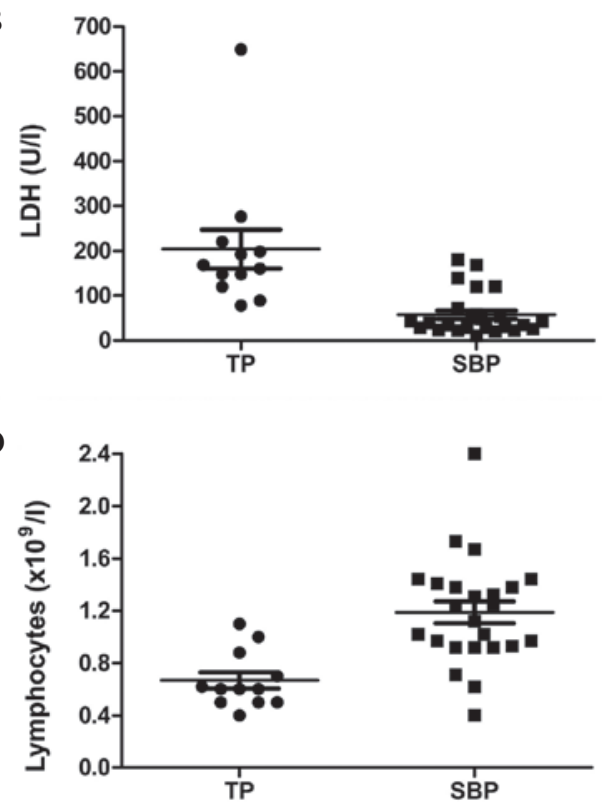

Figure 1. Ascitic and hematological observations of patients with TBP and SBP. The TBP group showed (A) higher protein concentrations, (B) higher LDH activity levels and (C) ADA activity levels in ascites and (D) a lower mean hematological lymphocyte cell count. TBP, tuberculous peritonitis; SBP, spontaneous bacterial peritonitis; $\mathrm{LDH}$, lactate dehydrogenase; ADA, adenosine deaminase.

total white cell count did not differ significantly between the two groups, but the lymphocyte population was significantly decreased in the TBP group when compared with the SBP group $\left(0.67 \pm 0.22 \times 10^{9} / 1\right.$ vs. $\left.1.19 \pm 0.41 \times 10^{9} / 1 ; \mathrm{P}<0.01\right)$. The serum levels of cancer antigen (CA)-125 in the two groups were elevated, but no significant difference was observed. The mean serum 
protein and albumin concentrations were significantly higher in the TBP group. In addition, the ascitic total white cell count was increased in the TBP group when compared with the SBP group, but no significant difference was observed. However, the proportion of white blood cells was statistically different with 11 cases $(92 \%)$ with lymphocytic predominance in the TBP group and 17 cases (68\%) with neutrophil predominance in the SBP group. Patients in the TBP group had significantly higher ascitic protein, adenosine deaminase (ADA) and lactate dehydrogenase (LDH) levels when compared with those in the SBP group, whereas the distribution of the serum ascites albumin gradient did not differ between the two groups. The ascitic protein level was $>25 \mathrm{~g} / 1$ in 9 patients $(75 \%)$ in the TBP group (range, 21.62-56.70 g/l) and 2 patients $(8 \%)$ in the SBP group (range, 1.50-34.80 g/1). Ascitic ADA activity levels were $>27 \mathrm{U} / 1$ in 8 patients $(67 \%)$ in the TBP group (range, 11-77 U/1), but no patients in the SBP group had levels $>27 \mathrm{U} / 1$ (range, 2-12 U/1). The ascitic LDH level was >90 U/1 in 10 patients $(83 \%)$ in the TBP group (range, 78-649 U/l) and 5 patients (20\%) in the SBP group (range, 11-180 U/1).

\section{Discussion}

The therapeutic techniques to treat TBP and SBP differ largely. TBP requires conservative quadruple antituberculosis treatment, while SBP requires empirical antimicrobial therapy. However, the diagnostic complications of TBP presents a technical hindrance for effective therapy for these patients.

TBP in patients with cirrhosis presents with nonspecific signs and symptoms, including abdominal distension, fever, abdominal pain and diarrhea, and hence mimics those of SBP. In the present study, it was identified that the clinical symptoms are similar between TBP and SBP in patients with cirrhosis. The onset of TBP is often insidious, even in patients with cirrhosis $(7,8)$. Consistent with a previous study, the median duration of symptoms prior to presentation was $>1$ month for cirrhotic patients with TBP (8). All patients were examined for signs of TB at additional sites and three cases $(25 \%)$ in the TBP group were diagnosed with pulmonary TB, but no cases were identified in the SBP group. Therefore, examination for $\mathrm{TB}$ at additional sites is important for diagnosing TBP.

Numerous studies have demonstrated that SBP occurs mainly in cirrhotic patients with Child-Pugh class C $(10,11)$, with only one study demonstrating that TBP occurs primarily in cirrhotic patients with Child-Pugh class B (8). The present study revealed similar results with 17 cases $(68 \%)$ of Child-Pugh class $\mathrm{C}$ in the SBP group and 8 cases $(67 \%)$ of Child-Pugh class B in the TBP group. The present results also indicate that compared with SBP, TBP may develop relatively early in the course of cirrhosis. In addition, the higher protein and albumin concentrations in the serum of cirrhotic patients with TBP may elucidate why the Child-Pugh class is mainly type $\mathrm{B}$ in the TBP group.

Notably, there was a significant decrease in the number of lymphocytes in the peripheral blood of cirrhotic patients with TBP. CD4+ T-lymphopenia is considered to be a reaction of mycobacterial infection and not a manifestation of underlying secondary immunodeficiency (12). Chau et al (13) hypothesized that sequestration of lymphocytes in the peritoneum may result in lymphopenia in the peripheral blood during a later phase of TBP. Therefore, lymphopenia in the peripheral blood may function as a marker for TBP.

Previous studies have shown that an elevation of serum CA-125 levels may be used as a novel marker for the diagnosis and follow-up of patients with TBP $(14,15)$. In the present study, the serum levels of CA-125 in the two groups were found to be elevated, however, no significant difference was observed. These results were not comparable with earlier observations since there have been no previous studies on the advantages of determining serum CA-125 levels for the diagnosis of TBP and SBP.

The predominance of lymphocytes in ascites is a characteristic of TBP (16). In the present study, 11 cases $(92 \%)$ were identified to have lymphocytic predominance in the TBP group, while 17 cases $(68 \%)$ had neutrophil predominance in the SBP group. Therefore, we hypothesized that the aforementioned ascitic fluid features may be a good indicator for diagnosis. In addition, patients in the TBP group were observed to have significantly higher ascitic fluid total protein levels when compared with the SBP group. Several studies have demonstrated that patients with an ascitic protein level of $>25 \mathrm{~g} / \mathrm{l}$ have a high sensitivity for TBP $(6,8)$. In the present study, the ascitic protein level was $>25 \mathrm{~g} / 1$ in 9 patients $(75 \%)$ in the TBP group, but only in 2 patients (8\%) in the SBP group. The protein concentration in the ascites of cirrhotic patients with SBP was $\sim 13 \mathrm{~g} / 1$ (17). Therefore, a higher protein concentration in the ascites may be considered as a useful marker for the diagnosis of TBP.

ADA has been investigated as a rapid diagnostic tool for TBP (18), however, the role of ADA in the setting of cirrhosis is controversial $(19,20)$. Hillebrand et al (19) identified that ADA activity showed imperfect specificity and low sensitivity in cirrhotic patients with TBP. These observations were countered by Liao et al (20), who reported that ADA activity showed a high specificity and sensitivity in those patients using a cut-off value of $>27 \mathrm{U} / 1$. In the present study, the mean ascitic ADA activity level in 12 patients with cirrhosis and TBP was $35.58 \mathrm{U} / 1$ and 8 of these patients had ADA activity level $>27 \mathrm{U} / 1$. The maximum ascitic ADA activity level in the 25 patients with SBP was $12 \mathrm{U} / 1$ with a mean of $4.32 \mathrm{U} / 1$. Therefore, we hypothesize that the examination of ADA activity is a critical test for diagnosing TBP.

Ascitic LDH levels increased due to the release of $\mathrm{LDH}$ from neutrophils (16). Elevation of ascitic LDH may be associated with numerous diseases, including TBP $(6,7)$ and SBP (21). A previous study has demonstrated that an ascitic LDH level of $>90 \mathrm{U} / 1$ is a useful parameter with high sensitivity and low specificity for the screening of TBP, irrespective of the presence of liver cirrhosis (6). In the present study, the mean ascitic LDH level in the TBP group was $204 \mathrm{U} / 1$ and 10 of these patients showed an LDH level of $>90 \mathrm{U} / 1$. The maximum ascetic LDH level in the SBP group was $180 \mathrm{U} / 1$ with a mean of $57 \mathrm{U} / \mathrm{l}$. Therefore, in a clinical setting, this parameter may be useful in discriminating against TBP.

It is important to be aware of the possibility of TBP in cirrhotic patients with ascites, including patients with known portal hypertension or SBP. In conclusion, clinical features and elevated serum CA-125 levels may not be specific in differentiating from SBP. However, TBP should be considered with the following criteria: Cirrhotic patients with Child Pugh 
class B; TB identified at additional sites; lymphopenia in the peripheral blood; an ascitic protein concentration of $>25 \mathrm{~g} / \mathrm{l}$; a predominance of lymphocytes in ascites; ascitic ADA activity levels of $>27 \mathrm{U} / 1$; and ascitic LDH levels of $>90 \mathrm{U} / 1$.

\section{Acknowledgements}

The study was supported by a grant from the Zhejiang Provincial Natural Science Foundation of China (no. LY12H03014) and the Zhejiang Province Health Bureau (no.2012KYA016).

\section{References}

1. Mehta JB, Dutt A, Harvill L and Mathews KM: Epidemiology of extrapulmonary tuberculosis. A comparative analysis with pre-AIDS era. Chest 99: 1134-1138, 1991.

2. Tang LC, Cho HK and Wong Taam VC: Atypical presentation of female genital tract tuberculosis. Eur J Obstet Gynecol Reprod Biol 17: 355-363, 1984.

3. Demir K, Okten A, Kaymakoglu S, et al: Tuberculous peritonitis - reports of 26 cases, detailing diagnostic and therapeutic problems. Eur J Gastroenterol Hepatol 13: 581-585, 2001.

4. Sochocky S: Tuberculous peritonitis. A review of 100 cases. Am Rev Respir Dis 95: 398-401, 1967.

5. Chow KM, Chow VC, Hung LC, Wong SM and Szeto CC: Tuberculous peritonitis-associated mortality is high among patients waiting for the results of mycobacterial cultures of ascitic fluid samples. Clin Infect Dis 35: 409-413, 2002.

6. Shakil AO, Korula J, Kanel GC, Murray NG and Reynolds TB Diagnostic features of tuberculous peritonitis in the absence and presence of chronic liver disease: a case control study. Am J Med 100: 179-185, 1996.

7. Aguado JM, Pons F, Casafont F, San Miguel G and Valle R: Tuberculous peritonitis: a study comparing cirrhotic and noncirrhotic patients. J Clin Gastroenterol 12: 550-554, 1990.

8. Kim NJ, Choo EJ, Kwak YG, et al: Tuberculous peritonitis in cirrhotic patients: comparison of spontaneous bacterial peritonitis caused by Escherichia coli with tuberculous peritonitis. Scand J Infect Dis 41: 852-856, 2009.

9. Rimola A, García-Tsao G, Navasa M, et al: Diagnosis, treatment and prophylaxis of spontaneous bacterial peritonitis: a consensus document. International Ascites Club. J Hepatol 32: 142-153, 2000 .
10. Choi JP, Lee SO, Kwon HH, et al: Clinical significance of spontaneous Aeromonas bacterial peritonitis in cirrhotic patients: a matched case-control study. Clin Infect Dis 47: 66-72, 2008.

11. Choi EJ, Lee HJ, Kim KO, et al: Association between acid suppressive therapy and spontaneous bacterial peritonitis in cirrhotic patients with ascites. Scand J Gastroenterol 46: 616-620, 2011.

12. Beck JS, Potts RC, Kardjito T and Grange JM: T4 lymphopenia in patients with active pulmonary tuberculosis. Clin Exp Immunol 60: 49-54, 1985.

13. Chau TN, Leung VK, Wong S, et al: Diagnostic challenges of tuberculosis peritonitis in patients with and without end-stage renal failure. Clin Infect Dis 45: e141-e146, 2007.

14. Mas MR, Cömert B, Sağlamkaya U, et al: CA-125; a new marker for diagnosis and follow-up of patients with tuberculous peritonitis. Dig Liver Dis 32: 595-597, 2000.

15. Choi CH, Kim CJ, Lee YY, et al: Peritoneal tuberculosis: a retrospective review of 20 cases and comparison with primary peritoneal carcinoma. Int J Gynecol Cancer 20: 798-803, 2010.

16. Sanai FM and Bzeizi KI: Systematic review: tuberculous peritonitis - presenting features, diagnostic strategies and treatment Aliment Pharmacol Ther 22: 685-700, 2005.

17. Shaw E, Castellote J, Santín M, et al: Clinical features and outcome of spontaneous bacterial peritonitis in HIV-infected cirrhotic patients: a case-control study. Eur J Clin Microbiol Infect Dis 25: 291-298, 2006.

18. Riquelme A, Calvo M, Salech F, et al: Value of adenosine deaminase (ADA) in ascitic fluid for the diagnosis of tuberculous peritonitis: a meta-analysis. J Clin Gastroenterol 40: 705-710, 2006.

19. Hillebrand DJ, Runyon BA, Yasmineh WG and Rynders GP: Ascitic fluid adenosine deaminase insensitivity in detecting tuberculous peritonitis in the United States. Hepatology 24: 1408-1412, 1996

20. Liao YJ, Wu CY, Lee SW, Lee CL, Yang SS, Chang CS and Lee TY: Adenosine deaminase activity in tuberculous peritonitis among patients with underlying liver cirrhosis. World J Gastroenterol 18: 5260-5265, 2012.

21. Sevinc A, Sari R and Fadillioglu E: The utility of lactate dehydrogenase isoenzyme pattern in the diagnostic evaluation of malignant and nonmalignant ascites. J Natl Med Assoc 97: 79-84, 2005. 